\title{
Adaptive Reduced Feedback Links for Distributed Power Allocation in Multicell MISO-OFDMA Networks
}

\author{
Berna Özbek, Didier Le Ruyet, and Mylene Pischella
}

\begin{abstract}
For multi-antenna Orthogonal Frequency-Division Multiple Access (OFDMA) based multicell networks, the channel state information (CSI) of all users is required to share among base stations in order to perform distributed power allocation. However, the amount of feedback increases with the number of users, base stations, subcarriers and antennas. Therefore, it is important to perform a selection at the user side to reduce the feedback load and the complexity of resource allocation. In this letter, we propose adaptive reduced feedback links by choosing the users based on their approximate signal to interference noise ratio (SINR) and their locations in the cell to satisfy users' rate constraints. We illustrate the performance results of reduced feedback links by employing distributed resource allocation with link adaptation.
\end{abstract}

Index Terms-Multicell networks,reduced feedback, power minimization, OFDMA, multiple antennas.

\section{INTRODUCTION}

$\mathbf{T}$ HE increasing demand for wireless multimedia has led to coordinated multicell transmission which can increase data rate and reduces outage in cellular systems by mitigating intercell interference (ICI). In coordinated multicell Orthogonal Frequency Division Multiple Access (OFDMA) networks, the optimal resource allocation (RA) requires to solve the problem of both the power and subcarrier allocation jointly in all considered cells, taking into account the multicell interference. RA can be performed to maximize the sum rate or to minimize the power consumption. In this letter, the power minimization problem is considered to satisfy the users' data rate constraints in multicell networks.

Margin-adaptive joint RA algorithms have been proposed for the downlink of multicell OFDMA systems in [1] by optimizing each subcarrier individually. In [2], the determination of power level for each user in each cell has been performed in a distributed way by considering both inter-cell interference and target data rate requirements for OFDMAbased multicell networks. In order to establish coordinated transmission, base station (BS) cooperation entails sharing channel state information (CSI) and/or precoders which determines the level of cooperation and the performance gains. Adaptive limited feedback schemes that select the transmission strategy depending on power level at cell-edge and the users' locations have been presented for single carrier multi-antenna multicell networks in [3]. A feedback load reduction technique has been examined in [4] based on thresholds for multicell

Manuscript received October 21, 2013. The associate editor coordinating the review of this letter and approving it for publication was S. Sun.

B. Özbek is with the Izmir Institute of Technology, Electrical and Electronics Eng. Dep., Izmir, Turkey (e-mail: bernaozbek@iyte.edu.tr).

D. Le Ruyet and M. Pischella are with CEDRIC/LAETITIA, CNAM, Paris, France (e-mail: \{didier.le_ruyet, mylene.pischella\}@cnam.fr).

Digital Object Identifier 10.1109/WCL.2013.120513.130743 networks. The reduced feedback link design that chooses the users based on their approximate signal to interference noise ratio (SINR) has been presented for single antenna OFDMA based multicell networks in [5]. The existing RA algorithms for multiple input single output (MISO)-OFDMA multicell networks that allocated more than one user per subcarrier have assumed that the CSI between all the users and serving and interfering BS is available at all BSs. This causes a high feedback load and a sophisticated resource allocation algorithm at the BSs.

In this letter, we propose adaptive reduced feedback links for MISO-OFDMA systems to reduce feedback link rate and complexity of the resource allocation by minimizing the total transmit power subject to per-user rate constraint. The proposed feedback link is performed by selecting the best clusters based on an approximate SINR rather than only channel gain. The proposed criterion is calculated at the receiver side by assuming that only one beam is employed for each cluster and assuming that the maximum interference is received from the other cells. We determine the number of clusters for each user according to their location and their rate constraints adaptively to reduce the feedback load. Then, each user selects the clusters and feeds back their CSI to its serving BS. The joint user scheduling and power allocation algorithm at the BS is performed in a distributed way to schedule more than one user at each cluster. We perform zero forcing (ZF) precoding to avoid inter-user interference (IUI) at each cell and power minimization to reduce the inter-cell interference (ICI) among the BSs.

\section{SYSTEM MODEL}

We consider a downlink MISO-OFDMA multicell system composed of $U$ base stations, each of which has $K$ users. Each BS has $N_{t}$ transmit antennas, and each user has one receive antenna. The clustering structure where adjacent subcarriers are grouped and only one representative value for each cluster is selected selected for CSI of serving and interfering BSs is used to reduce feedback information. The channel coefficient of a cluster is determined by $\mathbf{H}_{q}=\overline{\mathbf{H}}_{m}$ where $\overline{\mathbf{H}}_{m}$ is the $N_{t} \times 1$ channel vector for $m$ th subcarrier. The value of $m$ is determined by $m=(q-1) N_{Q}+\arg \min _{1 \leq i \leq N_{Q}}\left\{\left\|\overline{\mathbf{H}}_{(q-1) N_{q}+i}\right\|^{2}\right\}$ for each $q=1,2, \ldots, Q$ where $Q$ is the number of clusters, $N_{Q}=M / Q$ is the number of subcarriers in one cluster and $M$ is the number of subcarriers.

The power-allocation related notations are defined in the following. $p_{u}^{\max }$ denotes the total available power at BS $u$, $p_{u, q}$ represents the allocated power and $p_{u, q}^{\max }$ is the maximum allowed power level to cluster $q$ by BS $u .\left\{p_{u, q}\right\}_{u=1}^{U}$ is stacked into a $U \times 1$ vector $\mathbf{p}_{q}=\left[p_{1, q}, p_{2, q}, \ldots, p_{U, q}\right]^{T}$ and then 
the power allocation matrix $\mathbf{P}$ with $U \times Q$ is constructed. The cluster-allocation related notations are defined in the following. The binary variable $a_{u, q}^{k, b} ; b \in\left[1,2, \ldots, N_{t}\right]$ indicates that cluster $q$ is allocated to user $k$ for beam $b$ at BS $u$ if $a_{u, q}^{k, b}=1 .\left\{a_{u, q}^{k, b}\right\}_{q=1}^{Q}$ for each beam is stacked into the vector $\mathbf{a}_{u}^{k}=\left[a_{u, 1}^{k, 1}, \ldots, a_{u, 1}^{k, N_{t}}, \ldots, a_{u, Q}^{k, 1}, \ldots, a_{u, Q}^{k, N_{t}}\right]^{T}$ and then $\left\{\mathbf{a}_{u}^{k}\right\}_{k=1}^{K}$ is stacked to a matrix $\mathbf{A}_{u}$ column by column. Finally, a matrix $\mathbf{A}=\left[\mathbf{A}_{1}, \ldots, \mathbf{A}_{U}\right]$ indicates how the users are allocated to the clusters for all beams and all cells.

Considering that the optimization variables are the set of clusters $\mathbf{A}$ and the set of power values $\mathbf{P}$, the margin adaptive resource allocation problem is defined as:

$$
\min _{\mathbf{A}, \mathbf{P}} \sum_{u=1}^{U} \sum_{k=1}^{K} \sum_{q=1}^{Q} \sum_{b=1}^{N_{t}} a_{u, q}^{k, b} p_{u, q}^{b}
$$

subject to

$$
\begin{aligned}
R_{u}^{k} & \geq T_{u}^{k} \quad \forall k, \forall u \\
p_{u, q} & \leq p_{u, q}^{\max } \quad \forall u, \forall q \\
p_{u, q} & \geq 0 \quad \forall u, \forall q \\
\sum_{q=1}^{Q} p_{u, q} & \leq p_{u}^{\max } \quad \forall u \\
\sum_{k=1}^{K} a_{u, q}^{k, b} & \leq 1 \quad \forall u, \forall q, \forall b \\
a_{u, q}^{k, b} & \in\{0,1\} \quad \forall k, \forall u, \forall q, \forall b
\end{aligned}
$$

where $T_{u}^{k}$ is the target rate of user $k .\left(C_{2}\right),\left(C_{3}\right)$ and $\left(C_{4}\right)$ are the constraints for power allocation, $\left(C_{5}\right)$ and $\left(C_{6}\right)$ are the constraints for cluster allocation. In this work, it is assumed that $p_{u, q}$ is equally assigned to the beams such that $p_{u, q}^{b}=$ $p_{u, q} / N_{t}$.

The rate of user $k$ at $\mathrm{BS} u$ is calculated as,

$$
R_{u}^{k}=\sum_{q=1}^{Q} \sum_{b=1}^{N_{t}} a_{u, q}^{k, b} R_{u, q}^{k, b}\left(p_{u, q}\right)
$$

where $R_{u, q}^{k, b}$ is the rate of cluster $q$ when it is allocated to user $k$ at beam $b$ at BS $u$ and is calculated by:

$$
R_{u, q}^{k, b}\left(p_{u, q}\right)=\frac{B}{Q} \log _{2}\left(1+\Omega \gamma_{u, q}^{k, b}\left(p_{u, q}\right)\right)
$$

where $B$ is the total bandwidth and $\Omega$ is a constant gap that is chosen depending on the required bit error rate (BER).

The SINR, $\gamma_{u, q}^{k, b}\left(p_{u, q}\right)$, is determined by,

$$
\gamma_{u, q}^{k, b}\left(p_{u, q}\right)=\frac{\frac{p_{u, q}}{N_{t}}\left|\left(\mathbf{h}_{u, u, q}^{k}\right)^{H} \mathbf{w}_{u, q}^{b}\right|^{2}}{\frac{N_{0} B}{Q}+U_{u, q}^{k, b}+C_{u, q}^{k}}
$$

where $N_{0}$ is the power noise density and $\mathbf{h}_{u, u, q}^{k}=$ $\left[h_{u, u, q}^{k, 1}, \ldots, h_{u, u, q}^{k, N_{t}}\right]^{T}$ is the channel vector of user $k$ with the channel coefficient $h_{u, u, q}^{k, b}$ that belongs to user $k$ at BS $u$ for cluster $q$ from antenna $b$ in cell $u$ and includes path loss and multipath effect of the wireless channels. Note that if $u \neq v, h_{u, v, q}^{k, b}$ represents the channel coefficient of the cochannel interfering link from antenna $b$ of BS $v$ to the user $k$ located in cell $u$. It is assumed that the wireless channel between each BS-user pair remains static over a sufficiently long duration to perform RA.

To avoid IUI at each cell, the precoding matrix $\mathbf{W}_{u, q}$ is obtained by using ZF by,

$$
\mathbf{W}_{u, q}=\beta_{u, q}\left(\mathbf{H}_{u, q}\right)^{H}\left[\left(\mathbf{H}_{u, q}\right)\left(\mathbf{H}_{u, q}\right)^{H}\right]^{-1}
$$

where $\mathbf{H}_{u, q}=\left[\mathbf{h}_{u, u, q}^{1}, \ldots, \mathbf{h}_{u, u, q}^{K}\right]^{T}$ denotes the matrix of $K \times N_{t}$ consisting of the stacked channel vectors of the pair of assigned users for each cluster at each cell and $\beta_{u, q}$ is calculated by,

$$
\beta_{u, q}=\frac{1}{\sqrt{\operatorname{tr}\left[\left(\left(\mathbf{H}_{u, q}\right)\left(\mathbf{H}_{u, q}\right)^{H}\right)^{-1}\right]}}
$$

IUI is calculated by $U_{u, q}^{k, b}=\frac{p_{u, q}}{N_{t}} \sum_{j=1 ; j \neq b}^{N_{t}}\left|\left(\mathbf{h}_{u, u, q}^{k}\right)^{H} \mathbf{w}_{u, q}^{j}\right|^{2}$ and ICI is determined by $C_{u, q}^{k}=$ $\sum_{v=1, v \neq u}^{U} \frac{p_{v, q}}{N_{t}} \sum_{b=1}^{N_{t}}\left|\left(\mathbf{h}_{u, v, q}^{k}\right)^{H} \mathbf{w}_{v, q}^{b}\right|^{2}$ where $\mathbf{w}_{u, q}^{b}$ is the $b^{\text {th }}$ column of precoding matrix $\mathbf{W}_{u, q}$.

\section{THE PROPOSED REDUCED FEEDBACK LINKS}

In order to perform a selection of clusters at the user side, firstly we assume that full power is assigned to each BS and is shared equally between the clusters. Then, we design a precoding vector for each user at each cell supposing no cooperation between BSs. Since it is assumed that only one user is served at each cell, IUI does not occur. For interference coming from the other cells, we consider the worst case scenario such that the precoding vector of interfering BSs is determined by assuming that each user receives a maximum interference from the other cells.

The approximate SINR value of each user for each cluster in multicell network is calculated as:

$$
\tilde{\gamma}_{u, q}^{k}=\frac{\frac{p_{u}^{\max }}{Q}\left|\left(\mathbf{h}_{u, u, q}^{k}\right)^{H} \tilde{\mathbf{w}}_{u, q}^{k}\right|^{2}}{\frac{N_{0} B}{Q}+\tilde{C}_{u, q}^{k}} \quad \forall k, \forall u, \forall q
$$

where $\tilde{C}_{u, q}^{k}=\sum_{v=1, v \neq u}^{U} \frac{p_{v}^{\max }}{Q}\left|\left(\mathbf{h}_{u, v, q}^{k}\right)^{H} \tilde{\mathbf{w}}_{v, q}^{k}\right|^{2}$. In this case where only one user is assigned at each cell, the precoding vector is designed as $\tilde{\mathbf{w}}_{z, q}^{k}=\frac{\left(\mathbf{h}_{u, z, q}^{k}\right)^{H}}{\left\|\mathbf{h}_{u, z, q}^{k}\right\|}$ for $z=1,2, \ldots, U$.

At each cell, each user constructs independently a set $\mathbb{S}_{u}^{k}$ composed of the strongest $S_{u}^{k}$ clusters based on $\tilde{\gamma}_{u, q}^{k}$ values. Then, the CSIs of serving and interfering BSs of these selected clusters for each user are fed back and shared among BSs. In the following, we propose two algorithms to determinate the number of clusters, $S_{u}^{k}$ in each cell.

\section{A. Multicell Clustered S-Best criterion at fixed feedback rate}

In the first scheme, the number of strongest clusters for each user at each cell is fixed to $S_{u}^{k}=S$.

Let $\mathbb{T}_{u, q}$ be the set of users that feed back their CSI associated to the cluster $q$ at cell $u$ as,

$$
\mathbb{T}_{u, q}=\left\{k \in\{1,2, \ldots, K\}: q \in \mathbb{S}_{u}^{k}\right\}
$$

The $S$ value is defined as a function of the target rate and the number of users in the multicell networks. Consequently, total feedback load is proportional to $K S$ at each cell. 


\section{B. Multicell Clustered S-Best at adaptive feedback rate}

In the second scheme, the number of feedback clusters, $S_{u}^{k}$ is determined adaptively according not only to the target rate but also to the location of the users in the cell.

For link adaptation, the target SINR, $\Gamma_{u}^{k}$, is chosen from a set of predefined values according to the location of the users in the cell. Then, the number of target clusters, $G_{u}^{k}$, for $k^{\text {th }}$ user at cell $u$ is calculated by,

$$
G_{u}^{k}=\frac{T_{u}^{k}}{\frac{B}{Q} \log _{2}\left(1+\Omega \Gamma_{u}^{k}\right)}
$$

The number of feedback clusters by each user is determined based on the number of target clusters determined in Eq.(9). Since the corresponding $\Gamma_{u}^{k}$ is selected according to the location of the user, the farthest user requires more clusters than the nearest user to achieve the same target rate. Let's define a variable

$$
f_{u}^{k}=\left[e \frac{G_{u}^{k}}{Q}\right]_{1}^{+}
$$

where $[.]_{1}^{+}$denotes the orthogonal projection onto interval $[0,1]$ and $e$ is a fixed spectral efficiency parameter that adjusts the feedback link rate.

Then, each user generates a binomial random variable with parameters $Q$ and $f_{u}^{k}$ to determine its number of feedback clusters, $S_{u}^{k}$.

In this proposed scheme, the farthest user will feed back more clusters than the nearest user with the usage of $f_{u}^{k}$ which is also proportional to $G_{u}^{k}$ through $\Gamma_{u}^{k}$.

As described in the previous section, the set $\mathbb{S}_{u}^{k}$ which includes the best $S_{u}^{k}$ clusters of the user $k$ is obtained. Then, the set of $\mathbb{T}_{u, q}$ is constructed as given in Eq.(8).

\section{The distributed solution for MISO case}

It is assumed that the channel vectors $\mathbf{h}_{u, v, q}^{k} ; \forall u, v$ for $k \in \mathbb{T}_{u, q}$ belonging to each cluster at each cell is perfectly available at all BSs. Then, we perform the joint user scheduling and power allocation algorithm as follows:

- For $q=1$ to $Q$ :

- Step 1: Construct the user set denoted by $\mathbb{U}_{u, q}$ of size $\left(\begin{array}{c}\left|\mathbb{T}_{u, q}\right| \\ N_{t}\end{array}\right)$ at each cell individually by considering the users in the set of $\mathbb{T}_{u, q}$. Then, construct the user supersets called $\mathbb{M}_{q}$ of size $\prod_{u=1}^{U}\left|\mathbb{U}_{u, q}\right|$ for multicell networks with all combination of the user sets defined in $\mathbb{U}_{u, q}$.

- Step 2: For $i=1$ to $\left|\mathbb{M}_{q}\right|$ :

* Calculate the required transmitted power for each element of $\mathbb{M}_{q}$ as:

$$
\mathbf{v}(i)=\mathbf{B}(i)^{-1} \mathbf{z}(i)
$$

where $\mathbf{v}(i)=\left[\begin{array}{llll}\tilde{p}_{1, q}(i) & \tilde{p}_{2, q}(i) & \ldots & \tilde{p}_{U, q}(i)\end{array}\right]^{T}$, $\mathbf{z}(i)=\left[\begin{array}{llll}z_{1}(i) & z_{2}(i) & \ldots & z_{U}(i)\end{array}\right]^{T}$ and

$$
\mathbf{B}(i)=\left(\begin{array}{ccccc}
1 & -b_{12}(i) & -b_{13}(i) & \ldots & -b_{1 U}(i) \\
-b_{21}(i) & 1 & -b_{23}(i) & \ldots & -b_{2 U}(i) \\
-b_{31}(i) & -b_{32}(i) & 1 & \ldots & -b_{3 U}(i) \\
-\dot{\cdot}(i) & -b_{U 2}(i) & \ldots & \ldots & 1 \\
-b_{U 1}(i) & - & \ldots & & 1
\end{array}\right)
$$

with $z_{u}(i)=\frac{\left(N_{0} B / Q\right) \Gamma_{u}^{k}}{D_{u, q}(i)}$ and $b_{u v}(i)=$ $\frac{D_{v, q}(i) \Gamma_{u}^{k}}{D_{u, q}(i)} ; u \neq v$. The gains are calculated as,

$$
\begin{aligned}
& D_{u, q}(i)=\sum_{j=1 ; j \neq b}^{N_{t}}\left|\left(\mathbf{h}_{u, u, q}^{k}(i)\right)^{H} \mathbf{w}_{u, q}^{j}(i)\right|^{2} \\
& D_{v, q}(i)=\sum_{b=1}^{N_{t}}\left|\left(\mathbf{h}_{u, v, q}(i)\right)^{H} \mathbf{w}_{v, q}^{b}(i)\right|^{2} ; v \neq u
\end{aligned}
$$

* End.

- Step 3: Considering the constraints of $\left(C_{2}\right),\left(C_{3}\right)$ and $\left(C_{4}\right)$, the feasible power levels, $\mathbf{v}(i)$, at each BS for each $i$ is selected. Among the feasible power values, the user group that requires the minimum total transmitted power is selected as $i^{*}$.

$$
i^{*}=\arg \min _{i} \sum_{u=1}^{U} \tilde{p}_{u, q}(i)
$$

If none of the user group provides a feasible power level, only one BS transmits and the others are shut down. In that case, the BS which requires the minimum power is selected if this power level is feasible. Then, the users are allocated with the assigned power.

- Step 4: Check that each user from $i^{*}$ satisfies the rate constraint in $\left(C_{1}\right)$. If it does, remove this user from all sets $\mathbb{T}_{u, q}, \forall q$. Moreover, this user does not continue to feed back any information in the OFDMA frame.

- End.

All four steps described hereafter are performed at each BS independently. The proposed algorithm is consequently distributed, since no global controller is required to make the decision. The same decision is in fact taken by all BSs, based on the shared CSI available for all of them.

\section{Performance Results}

We obtain the performance results to illustrate the benefits of the reduced feedback links in multicell MISO-OFDMA networks with $N_{t}=2$ transmit antennas through wireless channels for $U=3$. The users are uniformly distributed in a multicell networks where a diameter of each cell is $600 \mathrm{~m}$. The transmitted power and the noise power density are set at $43.10 \mathrm{dBm}$ and $-174 \mathrm{dBm} / \mathrm{Hz}$ respectively. The path loss model is $L_{p}=128.1+37.6 \log _{10}(d(\mathrm{~km})) \mathrm{dB}$ and the wireless channel is modeled using $3 \mathrm{GPP}-\mathrm{TU}$ with a velocity of $30 \mathrm{~km} / \mathrm{h}$. The bandwidth, the carrier frequency and the number of clusters are selected as $5 \mathrm{MHz}, 2.4 \mathrm{GHz}$ and $Q=20$. The clusters are grouped into 12 subcarriers. Assuming the slot duration is $10 \mathrm{~ms}$, the feedback information is provided every $1 \mathrm{~ms}$. The target SINR values are chosen as $10.93 \mathrm{~dB}, 7.45 \mathrm{~dB}$ and $4.65 \mathrm{~dB}$ [7] to perform link adaptation according to the location of the user in the cell.

Firstly, the feedback load is calculated by averaging the number of users that feed back their CSI in one OFDMA frame. As shown in Fig.1(a), the proposed feedback link design with fixed rate reduces the feedback load up to $50 \%$ 

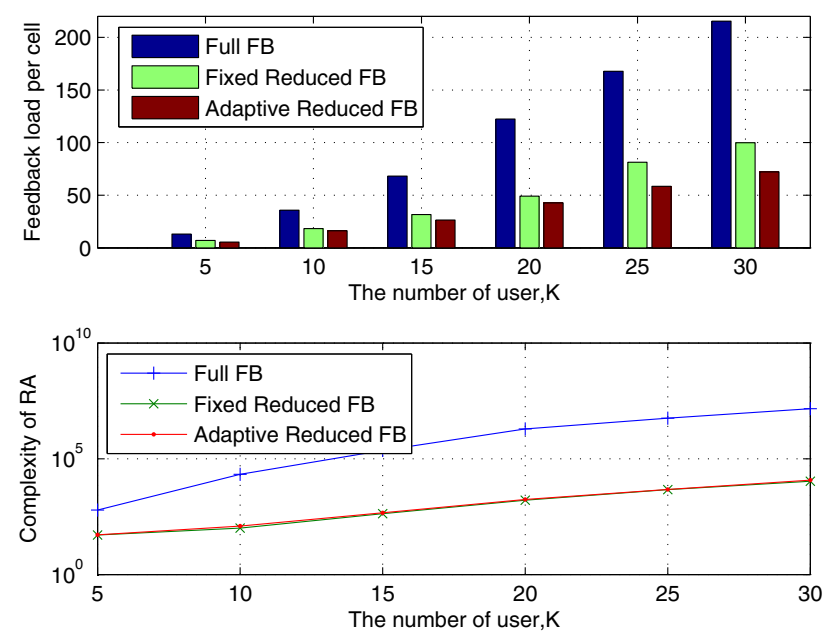

Fig. 1. (a) Feedback load; (b) complexity of RA vs. number of users in MISO-OFDMA multicell networks for $T=0.75 \mathrm{Mb} / \mathrm{s}$.

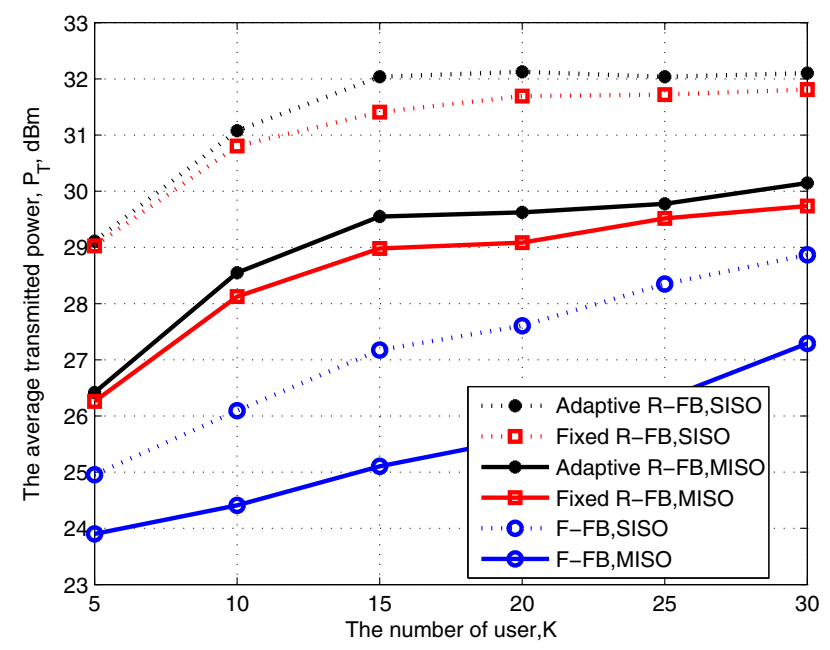

Fig. 2. Transmitted power vs. number of users in SISO/MISO-OFDMA multicell networks for $T=0.75 \mathrm{Mb} / \mathrm{s}$.

compared to full feedback when the number of users increases. In order to further reduce the feedback load, the adaptive rate scheme with link adaptation which provides additional $20 \%$ reduction has been proposed by taking into account both the location of the users and target rates. It is noted that the feedback load is averaged by taking into account the frame structure, which indicates that the feedback load is also changing in one OFDMA frame since the satisfied users do not continue to feed back any information to the BSs. Fur- thermore, as shown in Fig.1(b), the computational complexity in terms of number of operations during an OFDMA frame is reduced significantly compared to full feedback case.

Secondly, the comparison results in terms of total required transmitted power are illustrated in Fig.2. While obtaining these results, we set the number of feedback clusters in order to guarantee that at least $99 \%$ of the users achieve their data rate constraints at each frame. As shown in the performance evaluations, the total transmitted power is about $4 \mathrm{~dB}$ higher than with full feedback. This is however quite small compared to the achieved gain on the feedback link. It is also shown that the MISO-OFDMA case outperforms SISO-OFDMA case in multicell networks by requiring less transmitted power.

\section{CONCLUSION}

In this letter, we have examined the adaptive reduced feedback links for cooperative multicell MISO-OFDMA networks. Based on the approximate SINR values and the location information of the users in the cell, an adaptive reduced rate clustered S-best criterion has been proposed by employing link adaptation. As a result, the proposed reduced feedback links achieve the same performance than full feedback case in terms of user satisfaction ratio while the feedback load and the complexity of resource allocation has been significantly reduced.

\section{AcKnowledgment}

This research was supported by a Marie Curie Intra European Fellowship within the 7th European Community Framework Programme as a part of INTERCELL project with the contract number PIEF-GA-2009-255128.

\section{REFERENCES}

[1] A. Abrardo, A. Alessio, P. Detti, and M. Moretti, "Centralized radio resource allocation for OFDMA cellular systems," in Proc. 2007 IEEE Int. Conf. Communications, pp. 5738-5743.

[2] M. Pischella and J.-C. Belfiore, "Distributed resource allocation for rate constrained users in multi-cell OFDMA networks," IEEE Commun. Lett., vol. 12, pp. 250-252, Apr. 2008.

[3] J. Zhang and J. G. Andrews, "Adaptive spatial intercell interference cancellation in multicell wireless network," IEEE J. Sel. Areas Commun., vol. 28 , no. 9 , pp. 1455-1468, Dec. 2010.

[4] A. Papadogiannis, H. J. Bang, D. Gesbert, and E. Hardouin, "Efficient selective feedback design for multicell cooperative networks," IEEE Trans. Veh. Techol., vol. 60, no. 1, pp. 196-205, Jan. 2011.

[5] B. Ozbek, D. Le Ruyet, and M. Pischella "Reduced feedback links for power minimization in distributed multicell OFDMA networks," in Proc. 2012 IEEE International Communication Conference.

[6] P. Svedman, S. K. Wilson, L. J. Cimini, and B. Ottersten, "Opportunistic beamforming and scheduling for OFDMA systems," IEEE Trans. Commun., vol. 55, pp. 941-952, May 2007.

[7] A. Goldsmith and S. Chua, "Adaptive coded modulation for fading channels," IEEE Trans. Commun., vol. 46, no. 5, pp. 595-602, 1998. 